\title{
Carotid ligation: what happens in the long term?
}

\author{
AJAYA N JHA,* PAUL BUTLER, $\dagger$ RICHARD H LYE,* RICHARD A FAWCITT, $\dagger$ \\ From the University Department of Neurosurgery, ${ }^{*}$ Department of Neuroradiology, $\dagger$ Manchester Royal \\ Infirmary, Manchester, UK
}

SUMMARY The authors reviewed a consecutive series of 115 patients who underwent common carotid ligation during the period 1954-1984. Average follow-up was 10 years. Seventy-three (63\%) patients presented following a subarachnoid haemorrhage (SAH) and $42(36 \%)$ presented with the mass effect of an unruptured aneurysm. Thirty-nine (34\%) patients were lost to follow-up of whom 27 had had a previous SAH. Forty-six (63\%) of the 73 patients traced had suffered a SAH and amongst this group, 11 patients $(24 \%)$ died from a proven or suspected recurrent haemorrhage within 10 years of ligation. The fatal recurrent haemorrhage rate was, therefore, $2.4 \% /$ year. Thirty $(71 \%)$ of the 42 patients who presented with unruptured aneurysms were traced. Seven of these $(23 \%)$ died: two following haemorrhage, 1 year and 16 years after carotid ligation and three patients died as a direct consequence of carotid ligation. Check angiographic studies were available for 55 patients following carotid ligation, a mean of 8.4 years after the procedure. Thirteen were conventional angiograms and 42 were intravenous angiograms obtained using the digital subtraction technique. Seventy-six per cent of the aneurysms visualised on the initial studies were either smaller or had apparently disappeared. Only four new aneurysms were detected and in two of these instances, the initial angiographic studies had been incomplete. The authors conclude that the annual rate of fatal recurrent haemorrhage from an intracranial aneurysm following common carotid ligation is of a similar magnitude to that of the natural history of conservatively managed ruptured intracranial aneurysms. Moreover, carotid ligation apparently does not prevent haemorrhage from a previously unruptured aneurysm and the procedure appears to carry a significant morbidity and mortality, even in patients with an unruptured aneurysm.

Currently, the preferred neurosurgical management of the patient with an intracranial aneurysm requires the application of a clip across the neck of the aneurysm. There remain occasions, however, when ligation of the common carotid artery may be considered a suitable or preferable alternative. ${ }^{1}$ Several earlier studies have claimed to show that common carotid ligation benefits patients with aneurysms of the intracranial circulation. ${ }^{2-4}$ There have been few long term studies of patients who have undergone common carotid ligation as part of the treatment for an intracranial aneurysm. It has, however, been suggested that common carotid ligation may not afford long term protection against recurrent hae-

Address for reprint requests: Mr RH Lye, Department of Neurosurgery, Manchester Royal Infirmary, Manchester M13 OWL, UK.

Received 9 August 1985.

Accepted 20 October 1985 morrhage from an aneurysm. ${ }^{5}$ Since common carotid artery ligation remains part of the neurosurgical armamentarium, it is important to confirm or refute this claim. This study reviews the early and long term results of common carotid artery ligation in two groups of patients: those who had suffered a previous subarachnoid haemorrhage (SAH) and those who had presented with the effects of an unruptured intracranial aneurysm.

\section{Clinical materials and methods}

We reviewed the case histories of patients who underwent ligation of the ipsilateral common carotid artery as part of the management of an intracranial aneurysm(s) in our Department during the period 1954-1984. In all instances, the carotid ligation was achieved by gradual occlusion over a period of three to five days. Fourteen patients who underwent common carotid ligation as part of the management for other conditions were excluded from this study. An attempt was made to contact all patients and those who responded were examined, had a computed tomography 
(CT) scan and an angiographic study using the digital intravenous subtraction technique (DIVA). Informed consent was obtained from all patients, apart from eight who refused and therefore only underwent clinical examination. Attempts were made to trace the non-respondents (39) via their General Practitioner or the Family Practitioner Committee in order to ascertain their quality of life or the mode of death, if known. Only 13 patients had had conventional angiographic studies done prior to the advent of DIVA. These findings are included in this report and these patients did not have a further DIVA examination. An attempt was made to grade retrospectively the condition of each patient at the time of initial presentation, using the criteria defined by Hunt and Hess. ${ }^{6}$ In all cases, the pre-operative angiogram was compared with serial arterial or DIVA studies to determine changes in aneurysm size, vessel calibre and the appearance of new aneurysms.

\section{Summary of cases}

One hundred and fifteen patients underwent common carotid ligation in our Unit during the period 1954-1984 (table 1). Seventy-three $(63 \%)$ of these presented following a SAH and 42 patients $(36.5 \%)$ presented with the mass effect of an unruptured aneurysm. There were 16 cases of multiple aneurysms and six patients had giant aneurysms. Follow-up was complete in 76 patients $(66 \%) ; 26$ of these had died, the mean period of follow-up prior to death being six years. The mean follow up for the fifty survivors was 12 years and the mean follow up for the whole group was 10.3 years (range 2 weeks to 27 years). Amongst these 76 patients, 64 patients $(60 \%)$ had had a previous subarachnoid haemorrhage and $30(39 \%)$ had an unruptured aneurysm. Thirty-nine (31\%) patients were lost to follow-up after a mean of 3.75 years (range two weeks to 20 years) and half of these patients were followed for more than two years (fig 1). Information about their clinical condition at the time of their last attendance was available but their current state was unknown.

Review of the pre-operative angiograms demonstrated a total of 141 aneurysms with a preponderance of aneurysms in the vicinity of the posterior communicating artery (table 2). This was to be expected since carotid ligation has generally been recommended for aneurysms of the internal carotid artery. ${ }^{7}$ In the early part of the series, routine four vessel angiography was not performed and therefore the true proportion of multiple aneurysms in the series is probably an under-estimate. In 20 cases an anterior communicating artery aneurysm was demonstrated and in nine of these the blood supply was predominantly unilateral. The middle cerebral artery aneurysms (11) were generally part of the group of multiple aneurysms and were not primarily treated by carotid ligation.

\section{Early results}

Analysis of complications following carotid ligation with respect to clinical grading of the patient at the time of ligation is shown in table 3. Data were only available for 114 patients. Forty-two $(36.6 \%)$ had unruptured aneurysms and $19(45 \%)$ of these either new neurological deficits appeared or there was a worsening of pre-existing neurological deficits follow-
Table 1 Common carotid ligation 1954-1984

\begin{tabular}{lrl}
\hline Analysis of population & & \\
\hline 115 patients & M 34 & Mean 47 yrs. (22-73 yrs) \\
SAH & F 81 & Mean 52 yrs. (20-77 yrs) \\
& M 22 & \\
Cranial nerve palsy & F 51 & \\
M 12 & \\
Giant & F 30 & \\
Multiple (ipsilateral) & 6 & \\
$\quad$ (bilateral) & 14 & \\
\hline
\end{tabular}

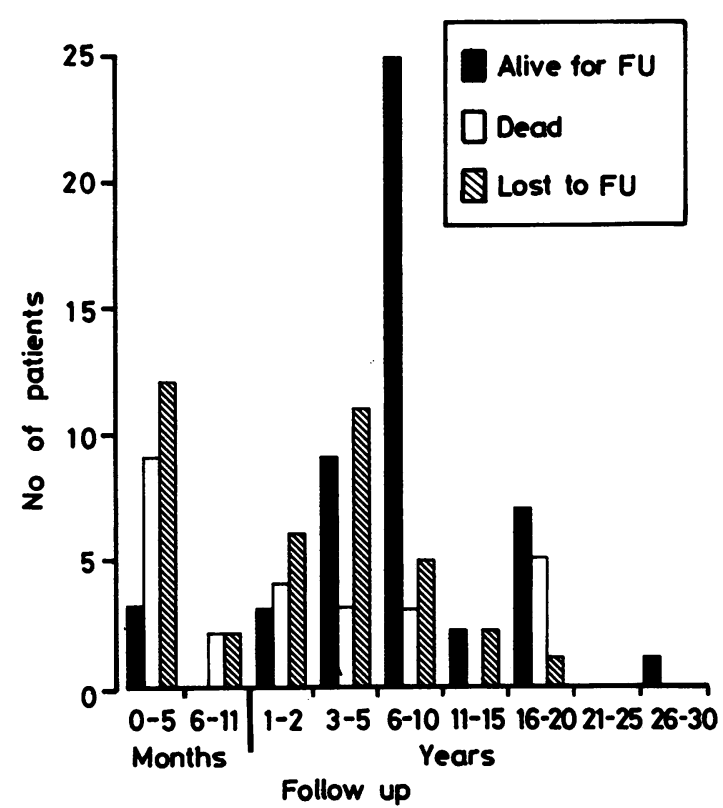

Fig 1 Follow-up for consecutive series of 115 patients undergoing common carotid ligation (ruptured and unruptured intracranial aneurysms).

Table 2 Aneurysm site (115 patients)

\begin{tabular}{ll}
\hline & Total No. of Aneurysms \\
\hline Posterior communicating & 65 \\
Other internal carotid & 45 \\
Anterior cerebral complex & $20(9)^{*}$ \\
Middle cerebral artery & 11 \\
\hline *No. with unilateral circulation &
\end{tabular}

ing ligation. Thirty-four (47\%) of 72 patients who had had a previous SAH developed additional permanent neurological deficits following carotid ligation. Transient neurological deficits included confusion, dysphasia, weakness of limbs and deterioration of vision. It can be seen from the table that the proportion of patients developing either transient or 
Table 3 Carotid ligation: complications $n=115^{*}$

\begin{tabular}{|c|c|c|c|c|c|}
\hline Grade of presentation & Unruptured & $I$ & $I I$ & $I I I$ & IV \\
\hline $\begin{array}{l}\text { Number } \\
\text { Neurological deficit }\end{array}$ & 42 & 18 & 33 & 19 & 2 \\
\hline $\begin{array}{l}\text { Transient } \\
\text { Permanent } \\
\text { Other complications }\end{array}$ & $\begin{array}{r}15 \\
19 \\
7\end{array}$ & $\begin{array}{l}6 \\
4 \\
5\end{array}$ & $\begin{array}{r}15 \\
15 \\
5\end{array}$ & $\begin{array}{r}8 \\
14 \\
7\end{array}$ & $\begin{array}{l}1 \\
1 \\
1\end{array}$ \\
\hline
\end{tabular}

*Data unavailable in one patient who is therefore excluded.

permanent neurological deficits increased when the clinical condition of the patient was poor. Of the 50 patients who had been traced and were still alive after carotid ligation, $39(78 \%)$ had an adequately documented blood pressure assessment at the initial admission. Amongst this group the average age was 50.6 years at the time of ligation and the mean follow up at the time of review was 8.1 years (range 3 months to 27 years). There was no observable difference in either the systolic or the diastolic blood pressures at the time of review compared to the initial assessment. However, 11 of the 39 patients $(36.7 \%)$ had become hypertensive at some stage and were currently receiving hypotensive therapy at the time of review.

A study of the natural history of untreated ruptured aneurysms ${ }^{8}$ has suggested that younger patients have a longer recurrent haemorrhage-free interval than older patients. We did not find this (fig. 2), there being an apparently random distribution between age at initial presentation and the interval to re-bleed.

Follow-up was completed in $76(66 \%)$ of all the patients. Forty-six $(60.5 \%)$ of these had had a previous subarachnoid haemorrhage and 30 (39.5\%) had presented with an unruptured aneurysm. Complete follow-up was defined as attendance at review or

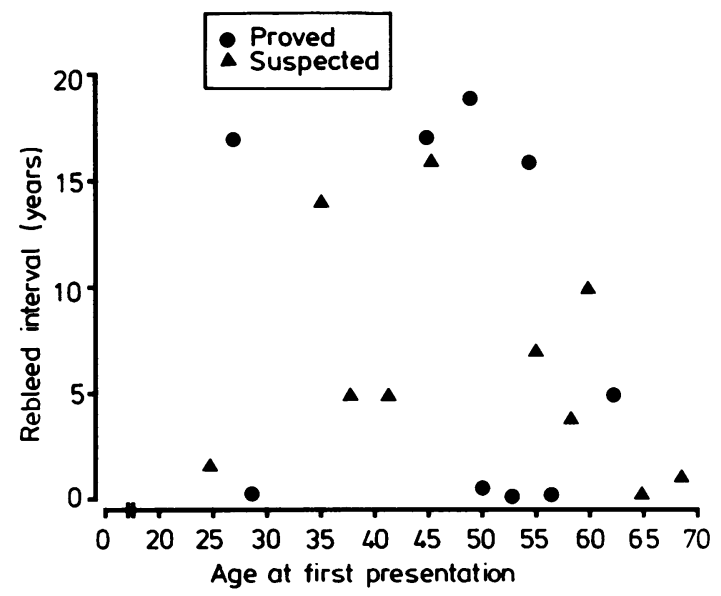

Fig 2 Interval of re-bleed from the time of initial presentation as a function of age at presentation (patients suffering a proven or suspected recurrent haemorrhage). definite evidence of the patient's demise. Twenty-six of these patients $(34 \%)$ had died, an overall mortality of $34.2 \%$ and an annual mortality rate of $3.4 \%$ (table 4).

Eleven of the 46 patients who had suffered a SAH in whom follow-up was completed had died of proven or suspected recurrent haemorrhage, yielding an annual rate for fatal recurrent haemorrhage of $2 \cdot 4 \%$. Two patients with previously unruptured aneurysms subsequently died of a suspected recurrent haemorrhage one year and sixteen years following carotid ligation. We defined "proven haemorrhage" as evidence of haemorrhage obtained at necropsy or haematoma visible on CT scans or a history highly suggestive of SAH in addition to confirmation of the presence of xanthochromia in the cerebrospinal fluid following lumbar puncture. "Suspected haemorrhage" was taken to be the cause in patients from whom a history typical of SAH was obtained or when there was certification that the cause of death was SAH or that the relatives of the deceased had been told that the cause of death was a "further haemorrhage". An anterior communicating artery aneurysm (14\% of all aneurysms for which carotid ligation was performed) was responsible for recurrent haemorrhage in $21 \%$ of those patients with recurrent SAH following carotid ligation. It is of interest that three of the seven documented late recurrent haemorrhages occurred after $\mathbf{1 5}$ years. Thirteen of the $\mathbf{2 6}$ $(50 \%)$ of the known deaths were a result of a proven or suspected SAH and two of these patients had previously presented with unruptured posterior communicating artery aneurysms. One died a year after the carotid ligation, the other 16 years following this procedure. Of the five deaths attributed to nonhaemorrhagic deterioration (three of these were in patients with unruptured aneurysms), the deterioration occurred in the post-operative phase. In both groups of patients, there were six deaths, the causes of which could not be ascertained.

Within the first six months following operation, eight patients died and in seven of these, the cause of death was either undetermined or attributable to cerebrovascular incidents. Three of these patients were thought to have died as a direct consequence of the operation and significantly these patients presented with painful third nerve palsies caused by an un- 
Table 4 Patient mortality

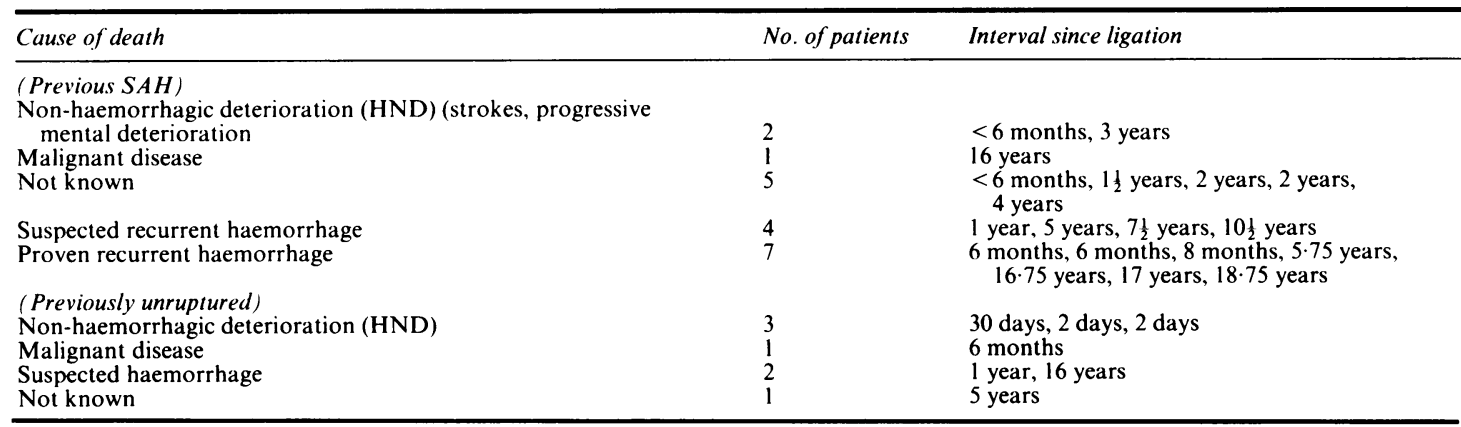

ruptured posterior communicating artery aneurysm. Their average age was $70 \cdot 3$ years. Four of the other deaths occurred in patients who had had a previous SAH and in two of these the cause of death was suspected or proven to be recurrent haemorrhage. During the same six month interval, 12 patients were lost to follow-up and it is possible that some of these may have died as a result of recurrent haemorrhage. (vide infra).

\section{Late results}

Twenty-seven of the 73 patients who underwent carotid ligation following SAH were lost to follow-up. Eleven of the remaining 46 died of a proven or suspected haemorrhage (total re-bleed rate of $2.4 \%$ per annum), two died of non-haemorrhagic deterioration, one died of a cerebrovascular incident and one patient died from malignant disease. In four patients, the cause of death was undetermined. If in addition to those deaths known or suspected to have followed recurrent haemorrhage, we assume that the five patients dying from unknown causes had in fact died following repeat $\mathrm{SAH}$, the annual fatal re-bleed rate after the first six months would increase to $3.5 \%$ for patients who undergo carotid ligation for an aneurysm which has already bled once.

Seven patients had died amongst the 30 patients who had had previously unruptured aneurysms and were subsequently traced. Therefore, the known mortality rate for this group was $23 \%$. Five of these seven patients died from cerebrovascular events. Thus the mortality from cerebrovascular events following ligation for an unruptured aneurysm was $16.7 \%$. It is probably significant that the average age of this group of patients who died following ligation for an unruptured aneurysm was 68 years, since it is likely that carotid ligation as opposed to intracranial obliteration of the aneurysm was the preferred treatment for the older patient $(68$ years $\simeq$ average life expectancy).
Post-ligation angiographic studies were available in 55 patients. In 13 of these, conventional angiograms were available and in 42 the latest radiological examination was a DIVA study. The final angiogram was obtained a mean of 8.4 years (range 2 weeks to 27 years) after the ligation (fig 3). At review, angiography revealed a total of 67 aneurysms and their distribution is shown in table 5. Comparison of pre- and post-operative angiograms in these 55 patients revealed that $51(76 \%)$ of the 67 aneurysms originally identified were either smaller or could not be involved. As can be seen from the table, this trend varied according to aneurysm site, $50 \%$ of the ante- $\frac{}{\Phi}$ rior communicating artery aneurysm remaining unchanged in size. Although four new aneurysms were observed to appear during the follow-up period, in two of these cases the pre-operative angiographic studies were incomplete. Three of the four newce. aneurysms occurred in patients who had undergone carotid ligation for an anterior communicating artery aneurysm. There was no evidence of re-canalisation of the ligated vessel, nor was there any angiographic

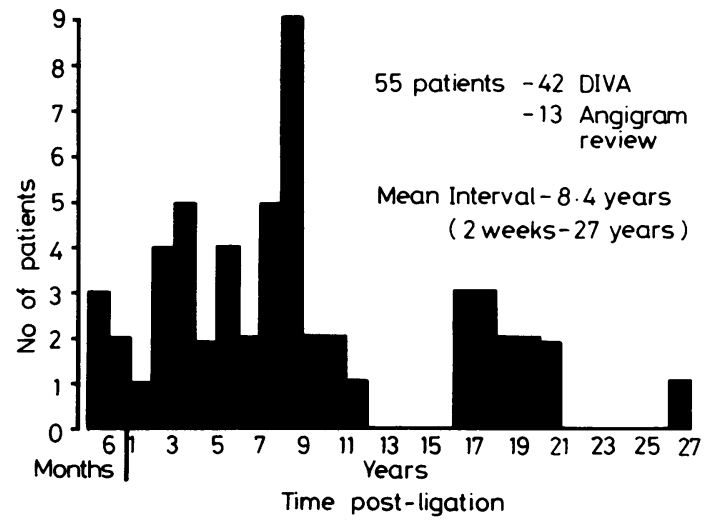

Fig 3 Timing of check angiography at post-operative review. 
Table 5 Radiological review

\begin{tabular}{cllcccc}
\hline Aneurysm & Number & Bigger & Same & Smaller & Not seen \\
\hline PCA & 36 & 1 & 8 & 5 & 22 \\
Other ICA & 20 & 0 & 2 & 5 & 1 \\
ACA & 10 & 0 & 5 & 1 & 0 & 13 \\
Total & 1 & 0 & 0 & 0 & 1 & 4 \\
\hline
\end{tabular}

Table 6 Annual rate of fatal recurrent haemorrhage*

\begin{tabular}{lll}
\hline & & Per Cent \\
\hline Carotid ligation & Winn et al 1982 & 1 \\
& Present series & $2 \cdot 4$ (proven) \\
& & $2 \cdot 2-5.9$ (possible) \\
Clipping of aneurysm & Shephard 1983 & $0 \cdot 1$ \\
\hline
\end{tabular}

*excludes deaths in first six months after initial presentation.

evidence of propagating thrombus since the ligated carotid artery appeared to fill retrogradely via the Circle of Willis in all cases. We did not observe enlargement of the contra-lateral carotid artery. This might have been expected to have occurred since there is experimental evidence of increased flow in the contralateral carotid artery following ligation. ${ }^{9}$ One patient experienced spontaneous thrombosis of the contra-lateral internal carotid artery and the Circle of Willis was fed by both vertebral arteries which appeared to have enlarged. This patient was asymptomatic. In all cases, where patent, the ipsilateral external carotid artery was observed to fill via collaterals from the contra-lateral side.

\section{Discussion}

There are limitations in the acquisition and interpretation of data in our study, as in all retrospective series. Nevertheless, certain conclusions appear to be valid. There have been several publications ${ }^{1479}$ claiming to show carotid ligation protects against recurrent haemorrhage. Most of these studies have concentrated on the short term results but there have been some long term studies suggesting that carotid ligation remains a valuable alternative treatment ${ }^{9}$ in the management of intracranial aneurysm. This view has been challenged. ${ }^{5}$ The long term natural history of ruptured aneurysms indicated an expected $2 \%$ mortality which is similar to that obtained using nonoperative treatment. ${ }^{28} \mathrm{~A}$ long term study ${ }^{5}$ of carotid ligation seems to imply that ligation is without any benefit and this has been our experience. Twenty-six per cent of our patients who had had a SAH died of a proven or suspected recurrent haemorrhage following ligation ( $2.4 \%$ per year). If we assume that those patients whose cause of death is unknown also died from a recurrent haemorrhage, then the annual rate of fatal re-bleeding increases to $3.5 \%$ per year. Further, if we speculate that those patients who had had a previous SAH followed by carotid ligation and were then lost to follow up be alive or dead, the mortality rates are reduced or increased respectively to $2 \cdot 2 \%$ per year and $5.9 \%$ per year.

Considering those patients who had previously unruptured aneurysms, the mortality rate either directly attributable to ligation or attributable to haemorrhage from the aneurysm subsequent to carotid ligation was $1.7 \%$ per year. Again, if we take into account the possible fates of patients lost to followup, the mortality rate may vary between $1.2 \%$ per year and $4.1 \%$ per year. The overall mortality rate for both groups of patients was $1.7 \%$ per year (minimum projected rate $1 \cdot 1 \%$, maximum projected rate $5.5 \%$ per year). These figures are of the same order of magnitude as those for conservatively managed aneurysms. Conversely, intracranial obliteration of the aneurysm yields results better to one order of magnitude. ${ }^{10}$

In the series presented, there was a preponderance of females, as has been found by previous authors. ${ }^{11}$ This seems to reflect the increased evidence of SAH and of aneurysms of the internal carotid artery in women. Carotid ligation has also been considered to be of value when treating aneurysms of the anterior circulation fed predominantly by one internal carotid artery. ${ }^{1}$ Our results suggest that this may be an overly optimistic approach since recurrent haemorrhage from this aneurysm site accounted for $21 \%$ of such episodes after carotid ligation.

It has been claimed that the procedure of common carotid ligation has two virtually opposed objectives: that of reducing the pressure head at the site of the aneurysm whilst at the same time avoiding neurological sequelae. ${ }^{12} \mathrm{We}$ were disturbed to find the very high morbidity $(45 \%)$ for carotid ligation as part of the treatment of unruptured aneurysms, although this may be a reflection of the fact that patients were not pre-selected for this procedure on the basis of preoperative tests. ${ }^{13}$ Not surprisingly, amongst those patients who had had a SAH the poorer the patient's clinical condition the poorer the outcome (table 3). Even carotid ligation for an unruptured aneurysm was associated with a significant mortality $(16 \cdot 7 \%)$ 
and two patients died from a SAH one and 16 years following ligation as part of the treatment for an unruptured aneurysm.

There have been several angiographic studies of both the natural history of aneurysms and of those aneurysms treated by carotid ligation. ${ }^{14-16}$ In one study in which follow-up was continued at intervals extending to 11 years following ligation, $77 \%$ of those aneurysms could not be seen. ${ }^{1}$ We did not consider it justifiable to carry out arterial angiograms on all our review patients because of the implied morbidity of this procedure. ${ }^{17}$ The advent of digital subtraction angiography which can be achieved using intravenous contrast media ${ }^{18}$ has to some extent circumvented this objection. It is possible to visualise intracranial aneurysms using DIVA studies although the definition is inferior to that of conventional angiography. Inevitably, some aneurysms will be missed using this technique but this implied that our findings are in fact biased in favour of a beneficial effect of carotid ligation on aneurysmal size. It is of interest that $72 \%$ of our aneurysms were either smaller or could not be seen following carotid ligation and this figure is similar to that report in another series using conventional angiography. ${ }^{1}$ However, failure to visualise an aneurysm sac does not imply that the aneurysm will not bleed again. ${ }^{8}$

\section{Conclusion}

Our study implies that radiologically there is some evidence of a beneficial effect following ligation of the common carotid artery in that a considerable proportion of aneurysms decrease in size or even disappear. Unfortunately it is clear that ligation of the carotid artery carries a considerable risk of both morbidity and mortality, especially in the older patient. This risk is not confined to patients whose aneurysm has previously ruptured. Furthermore, intact aneurysms may rupture many years after carotid ligation. Notwithstanding the encouraging radiological findings, the clinical results suggest that common carotid ligation does not reduce the risk of fatal recurrent haemorrhage in the long term when compared with the natural history of surgically untreated intracranial aneurysms.

We thank Miss Pamela Brown for typing the manuscript and the staff of the Departments of Neuroradiology and Neurosurgery.

\section{References}

${ }^{1}$ Tindall GT, Fleischer AS. Management of aneurysms of anterior circulation by carotid artery occlusion. In: Youmans JR. ed. Neurological Surgery. 2nd ed. Philadelphia: WB Saunders Co. 1982:1697-714.
${ }^{2}$ McKissock W, Richardson A, Walsh L. Posterior communicating aneurysms. A controlled trial of the conservative and surgical treatment of ruptured aneurysms of the internal carotid artery at or near the point of origin of the posterior communicating artery. Lancet 1960; 1:1203-8.

${ }^{3}$ Poppen JL, Fager CA. Intracranial aneurysms. Results of surgical treatment. $J$ Neurosurg 1960;17:283-96.

${ }^{4}$ Tindall GT, Odom GL. Treatment of intracranial aneurysms by proximal carotid ligation. In: Krayenbuhl H, et al. eds. Progress in Neurological Surgery, Vol. 3. Chicago: Year Book Medical Publishers 1969: 66-114.

${ }^{5}$ Winn HR, Richardson AE, Jane JA. Late morbidity and mortality of common carotid ligation for posterior communicating aneurysms. J Neurosurg 1977;47: 727-36.

${ }^{6}$ Hunt WE, Hess RM. Surgical risk as related to time of intervention in the repair of intracranial aneurysms. J Neurosurg 1968;28:14-20.

${ }^{7}$ Nishioka H. Results of the treatment of intracranial aneurysms by occlusion of the carotid artery in the neck: report on the cooperative study of intracranial aneurysms and subarachnoid haemorrhage. J Neurosurg 1966;25:660-82.

${ }^{8}$ Winn HR, Richardson AE, Jane JA. The natural history of cerebral aneurysms: the rate of late rebleeding. In: Brock M, ed. Modern Neurosurgery, Vol. 1 Heidelberg: Springer 1982:417-23.

${ }^{9}$ Symon L, Ishikane S, Lang S, Meyer JS. Quantitative measurement of cephalic blood flow in the monkey. A Study of vascular occlusion in the neck using electromagnetic flow. J Neurosurg 1963;20:198-218.

${ }^{10}$ Shephard RH. Ruptured cerebral aneurysms: early and late prognosis after surgical treatment. A personal series 1958-1968. J Neurosurg 1983;59:6-15.

${ }^{11}$ Locksley HB. Report on the cooperative study of intracranial aneurysms and subarachnoid haemorrhage. Section 5 part 1 . Natural history of subarachnoid haemorrhage, intracranial aneurysms and arteriovenous malformations. J Neurosurg 1966;25:219-37.

${ }^{12}$ Stephens WE. Pathology of the Cerebral Blood Flow Vessels. Saint Louis: CV Mosley, 1972:351-470.

${ }^{13}$ Leech PJ, Miller JD, Fitch W, Baker J. Cerebral blood flow, internal carotid artery pressure and the EEG as a field to the safety of carotid ligation. $J$ Neurol Neurosurg Psychiatry 1974;37:854-62.

14 Bjorkensten G, Troupp $H$. Changes in the size of intracranial arterial aneurysms. $J$ Neurosurg 1962;19:583-8.

${ }^{15}$ Somach FM, Shenkin HA. Angiographic end-results of carotid ligation in the treatment of carotid aneurysm. $J$ Neurosurg 1966;24:966-74.

${ }^{16}$ Tindall GT, Gorce JA, Lee JF, et al. Effect of common carotid ligation on size of internal carotid aneurysms and distal intra-carotid and retinal artery pressure. $J$ Neurosurg 1966;25:503-11.

${ }^{17}$ Nibbelink DW, Torner J, Henderson WG. Intracranial aneurysms and subarachnoid haemorrhage. Stroke 1977;8:202-18.

${ }^{18}$ Christenson PC, Ouitt TW, Fisher HD III, Nudleman, Rontrig H. Intravenous angiography using digital video subtraction: Intravenous certico-cerebrovascular angiography. Am J Neur Rad 1980;1:379-86. 